\title{
Prevalence of Salmonella serotypes and antibiogram of Salmonella typhi in a Tertiary Care Hospital in NCR Region, India
}

\author{
Ruchi Girotra $^{1 *}$, Reetika Dawar ${ }^{2}$, Ruby naz $^{1}$, Sunil Garg $^{3}$ and Rakesh Gupta ${ }^{3}$ \\ ${ }^{1}$ Department of Microbiology, Shaheed Hasan Khan Mewati, Government Medical College \\ Nalhar (Mewat) Haryana \\ ${ }^{2}$ Department of Microbiology, Indraprastha Apollo Hospital \\ ${ }^{3}$ Department of Internal Medicine and Critical Care, Sarvodaya Hospital and Research Centre, \\ YMCA Road,Sector 8 Near ESI Hospital Faridad-Delhi/NCR 121006 \\ *Corresponding author
}

\section{Keywords}

Salmonella typhi Salmonella paratyphi A, Enteric fever, Resistance.

\begin{tabular}{l} 
Article Info \\
\hline Accepted: \\
24 March 2016 \\
Available Online: \\
10 April 2016
\end{tabular}

\section{A B S T R A C T}

Salmonella typhi has been the most common aetiological agent of enteric fever syndrome while $S$. paratyphi A is responsible for only a minority of enteric fever cases. Salmonella typhi has also rapidly gained resistance to antibiotics like ampicillin, chloramphenicol and cotrimoxazole, and also to previously efficacious drugs like ciprofloxacin.It is imperative to know the current prevalence of Salmonella serotypes in order to understand vaccination strategy for typhoid. Also knowledge about the resistance pattern of the most predominant serotype is desirous for prescribing antimicrobials. 187 Blood culture specimens were received in BACTEC 9050 Bottles between January 2012 to January 2013 and presumptive isolates identified biochemically and confirmed by slide agglutination antiserum . Antibiotic susceptibility testing was performed by the Kirby Bauer disk diffusion method to the following antibiotics were put $\mathrm{up}(\mu \mathrm{g})$ : levofloxacin (5), nalidixic acid (30), ciprofloxacin (5), cefepime (30), cefotaxime(30)/ ceftriaxone (30)and azithromycin(15) (Hi-Media Laboratories Ltd, Mumbai). In addition first line antimicrobials like chloramphenicol(30), ampicillin (10), trimethoprim/sulfamethoxazole (25) were also put up. Out of 187 blood cultures, 30 Salmonella typhi and 8 Salmonella paratyphi were identified. The Salmonella typhi were resistant to the antimicrobials as follows : Ampicillin 66.7\%, chloramphenicol $10.0 \%$, cotrimoxazole $26.7 \%$,cefixime $9.75 \%$,cefepime $8.32 \%$,cefotaxime/ceftriaxone $10.32 \%$, ,levofloxacin $4.1 \%$, ciprofloxacin 13.3\%,Nalidixic acid $96.7 \%$ and Azithromycin 14.2\%,e presence of MDR (i.e resistance to ampicillin, chloramphenicol and co-trimoxazole) was $10 \%$. The evidence suggests that Salmonella typhi is still the predominant serotype followed by Salmonella paratyphi A with the rates of isolation of latter being more or less constant over the past decade. The evidence also suggests that the fluoroquinolones are the optimal choice for the treatment of typhoid fever in adults and that they may also be used in children inspite of resistance to nalidixic acid. The use of ceftriaxone and other third generation cephalosporins should be used only in case of non responsiveness to fluoroquinolones. 


\section{Introduction}

The enteric fever syndrome is an acute systemic illness characterised by fever headache and abdominal discomfort. Salmonella typhi is the most common aetiological agent, while $S$. paratyphi $A$ is responsible for only a minority of enteric fever cases and is traditionally associated with asymptomatic and/or mild clinical illness 1,2 .

Uncomplicated Acute typhoid fever is characterized by prolonged fever, disturbances of bowel function (constipation in adults, diarrhoea in children), headache, malaise and anorexia. Bronchitic cough is common in the early stage of the illness.(WHO). However, up to $10 \%$ of typhoid patients may develop serious complications like the presence of occult blood in the stool of $10-20 \%$ of patients, up to $3 \%$ may have melena, Intestinal perforation in up to $3 \%$ of hospitalized cases. 14. S. paratyphi $A$ is the causal agent for a substantial proportion of enteric fever episodes that cannot be distinguished clinically from typhoid fever episodes. While similar treatment strategies may work for both organisms, future enteric fever prevention strategies in Asia must focus on S. paratyphi $A$ as well as on S. typhi. ${ }^{29-31}$

Also Salmonella typhi has rapidly gained resistance to antibiotics like ampicillin, chloramphenicol and cotrimoxazole, and also to previously efficacious drugs like ciprofloxacin. $^{3,4 .}$

The emergence of antimicrobial resistance, especially the multidrug resistance to ampicillin, chloramphenicol and cotrimoxazole, has further complicated the treatment and management of enteric fever 5,6. In India, antibiotic resistance among $S$. typhi has been reported since 1960, and the first outbreak of multidrug resistant S. typhi (MDRST) was reported in Calicut ${ }^{7}$

As a result of the widespread dissemination of such strains, chloramphenicol can no longer be regarded as the first-line drug for typhoid fever caused due to S.Typhi. Although many strains remain fully sensitive to all quinolones, decreased susceptibility has been reported in many countries. ${ }^{8}$.Such isolates although apparently susceptible to ciprofloxacin on disc resting often show in vitro resistance to Nalidixic acid and are associated with increased clinical failure rates when treated with ciprofloxacin. ${ }^{9}$

The incidence of multidrug resistant (MDR) S. typhi was reported to be as high as 60 per cent while there are reports noting a decline ${ }^{11-13}$. Given the variation in the susceptibility patterns reported for S.typhi, it is important to constantly monitor it so as to provide suitable guidelines for treatment. Secondly in recent years, some changes in the epidemiology of enteric fever has been noted by various workers as there has been a rising incidence of $S$. paratyphi $A$ infections. ${ }^{25,26}$ This study was undertaken to understand the prevalence of Salmonella serotypes and antimicrobial profile of Salmonella typhi in a tertiary care hospital This is a first study of its kind to report the prevalence of serotypes and resistance pattern of Salmonella typhi including for azithromycin in NCR region and therefore its significance in choice of preventive strategy, antimicrobials and their judicious use in typhoid treatment.

\section{Materials and Methods}

Blood culture was done for 187 patients suspected to have enteric fever at Sarvodaya Hospital and Research centre, Faridabad during January 2012 to February 2013. 


\section{Inclusion Criteria}

The study included blood cultures of patients suspected of Salmonella septicaemia at Department of Microbiology of Sarvodaya Hospital and research centre. Only one isolate per patient were included in the study.

\section{Exclusion Criteria}

Patient who had received antibiotics in the last 7 days.

\section{Blood Culture}

Blood culture was carried out in continuous monitoring Automated blood culture system (BACTEC-9050).

Blood for culture was received in labeled BACTEC-9050 bottles (Becton Dickinson and Company).

The culture plates of positive specimens were incubated on Blood and Mac-Konkey agar at $37{ }^{\circ} \mathrm{C}$ for $18-24$ hours. Growth of organism and colony characters were noted after incubation.

Presumptive Isolates (non lactose fermenters) were identified biochemically and confirmed by slide agglutination, first with Salmonella polyvalent $\mathrm{H}$ and $\mathrm{O}$ followed by individual salmonella group antisera.

Escherichia coli ATCC 25922 was used as a quality control strain.

Commercially available six $\mathrm{mm}$ disks (Himedia Laboratories, Mumbai) were used. Antibiotic susceptibility testing was performed by the Kirby Bauer disk diffusion method to the following antimicrobials $(\mu \mathrm{g})$ :, levofloxacin(5), nalidixic acid (30), ciprofloxacin (5) azithromycin(15), cefepime (30), cefotaxime (30)/ ceftriaxone (30) (Hi-Media Laboratories Ltd, Mumbai). The Following previous first-line antimicrobials to which the strains could be resistant chloramphenicol (30), ampicillin (10), trimethoprim/ sulfamethoxazole (25) were also put up. For disk diffusion, test a maximum of 5 disks on a $100-\mathrm{mm}$ plate were put up. Diameter of the zones of complete inhibition were measured, including the diameter of the disk.

The disk strengths and zone size interpretation was in accordance with Clinical Laboratory Standards Institute (CLSI) M100 S23.

WHO guidelines for diagnosis, prevention and treatment of enteric fever suggests that Azithromycin disc test results should be interpreted with caution as the appropriate break-point recommendations for azithromycin against $S$. typhi are still not clear. However since on discussion with clinicians, patients responded satisfactorily to azithromycin even if isolates were intermediate according to current guidelines, we included azithromycin in the present study.

All those isolates testing resistant to nalidixic acid but sensitive to other quinolones in disc sensitivity were reported as sensitive. However a note was added stating that due to nalidixic acid resistance other quinolone even though sensitive in vitro testing could result in clinical failures.

Multi Drug resistance was defined as resistance to antibiotics such as chloramphenicol, ampicillin and trimethoprim-sulfamethoxazole (MDR strains). 


\section{Results and Discussion}

Out of 187 Blood samples followed up in the microbiology laboratory, 38 Salmonella species were identified. Salmonella typhi were the predominant type followed by Salmonella paratyphi A (Table 1). No other Salmonella serotype were detected during this period.

The Salmonella typhi were resistant to the antimicrobials as follows. Ampicillin 66.7\%, chloramphenicol 10.0\%, cotrimoxazole $26.7 \%$, cefixime $9.75 \%$, cefepime $8.32 \%$, cefotaxime/ceftriaxone $\quad 10.32 \%$, levofloxacin $4.1 \%$, ciprofloxacin $13.3 \%$, Nalidixic acid $96.7 \%$ and Azithromycin $14.2 \%$. Highest resistance was reported for nalidxic acid followed by ampicillin.

Four isolates $(10 \%)$ out of a total 42 Salmonella typhi, identified were found to be MDR. MDR isolates were mainly resistant to three antibiotics: ampicillin, chloramphenicol and cotrimoxazole.

In the current study, $S$. typhi and $S$. paratyphi $A$ were responsible for a total of $78 \%$ and $22 \%$ of cases of enteric fever respectively. This is consistent with a similar study carried out in Delhi in which $S$. typhi and $S$. paratyphi $A$ were responsible for a total of $79.7 \%$ and $20.3 \%$ of cases of enteric fever respectively in 1999. ${ }^{27}$ Another published study from North India was in concordance with our study where the proportion of $S$. paratyphi $A$ isolated from enteric fever cases remained almost constant throughout the study period from 1997 to 2001 . $^{28}$

In a study done in multiple countries in 2002-03, for Salmonella paratyphi A rates in Asia, $24 \%$ of enteric fever episodes in India were caused by $S$. paratyphi $A$. The study shows that $S$. paratyphi A rates in India have more or less remained stable over the past decade. However they are still a significant percentage of the total number of Salmonella serotypes.

Therefore the current rates of isolation of Salmonella serotypes in our study compared to previous studies done in 2012 has implications in current vaccination strategy in India. Licensed typhoid fever vaccines (Vi polysaccharide and live oral Ty21a) do not protect against infections caused by $S$. paratyphi $A$, and they may become less useful in controlling enteric fever in regions of India as consistent rates of Salmonella paratyphi A isolation between 20-30\% indicates vaccination strategies including bivalent vaccines that protect against $S$. typhi as well as $S$. paratyphi $A$ are still the recommended strategy for prevention of typhoid.Also since Salmonella paratyphi B vaccine is not still prevalent it is not recommended for immunization in India.

Wherever possible the treatment of enteric fever should be based on individual or local antibiotic susceptibility pattern. With decreased usage chloramphenicol resistance rates have fallen in India and Bangladesh and many isolates are once again susceptible to this drug. ${ }^{13}$

Resistance to chloramphenicol in our study was reported as $10.5 \%$ therefore could be suggested as choice for treatment of typhoid. However Chloramphenicol has several disadvantages of use including agranulocytosis of 1per 10,000 patients, a relatively high rate of relapse (5-7\%), long treatment courses (14 days) and the frequent development of a carrier state in adults. 14

Resistance to the fluoroquinolones may be total or partial. The so-called nalidixic-acidresistant $S$. typhi (NARST) is a marker of reduced susceptibility to fluoroquinolones compared with nalidixic-acid-sensitive strains. Nalidixic acid susceptibility has 
been validated as a screening test for reduced susceptibility to ciprofloxacin ${ }^{14}$ As in our study the Nalidixic acid resistance was $95.5 \%$ Even though ciprofloxacin sensitivity was $13.3 \%$ and levofloxacin $4.1 \%$. Therefore inspite of low resistance of major fluoroquinolone drugs,quinolones should be used cautiously in view of widespread resistance to nalidixic acid.

The fluoroquinolones are widely regarded as optimal for the treatment of typhoid fever in adults However, the emergence of MDR strains has reduced the choice of antibiotics in many areas.

It is therefore suggested that for nalidixicacid-sensitive $S$. typhi, seven-day regimens have proved highly effective and should be advised by clinicians For nalidixic-acidresistant infections a minimum of seven days of treatment at the maximum permitted dosage is necessary and10- 14 days are usually required. Courses shorter than seven days are unsatisfactory. ${ }^{14}$

The fluoroquinolones attain excellent tissue penetration, kill S. typhi in its intracellular stationary stage in monocytes/macrophages and achieve higher active drug levels in the gall bladder than other drugs. They produce a rapid therapeutic response, i.e. clearance of fever and symptoms in three to five days, and very low rates of post-treatment carriage.

Resistance to Ampicillin in our study was $61.2 \%$ of isolates being resistant to the drug. Therefore among the first line drugs, Salmonella typhi still has high resistance to ampicillin.

The resistance to 3rd Generation cephalosporins like cefixime (9.75), ceftriaxone/ cefotaxime(10.3) and $4^{\text {th }}$ generation cephalosporin, cefepime (8.32) is similar to a study which recorded resistance to ceftriaxone. ${ }^{15}$

Of the third-generation cephalosporins, oral cefixime has been widely used in children in a variety of geographical settings and found to be satisfactory. ${ }^{16,17,}{ }^{18}$ In our study, Salmonella typhi had low resistance to3rd and $4^{\text {th }}$ generation cepahalosprins. However resistance was higher than a study done department of microbiology JIPMER in on Current pattern in antimicrobial susceptibility of Salmonella typhi isolates from 2002-2003, where no resistance was reported. ${ }^{19}$

A trial of cefixime in MDR typhoid in Viet Nam indicated significantly higher treatment failure rates than with ofloxacin. ${ }^{20}$ Also the importance of $3^{\text {rd }}$ generation cephalosporin is emphasized in pregnant women and therefore there use be judicious. The antimicrobials generally recommended in pregnancy are the beta-lactams. However due to the increased resistance their use has become limited in developing countries.

The resistance to Azithromycin was $14.2 \%$ in our study. Azithromycin in a dose of 500 $\mathrm{mg}(10 \mathrm{mg} / \mathrm{kg})$ given once daily for seven days has proved effective in the treatment of typhoid fever in adults and children with defervescence times similar to those reported for chloramphenicol. ${ }^{21}$

The emergence of MDR $S$. typhi has again been noted with an incidence as high as $60 \%$ although there are other reports noting a decline [19].In our study a declining trend was noted with $10 \%$ of the isolates being multi drug resistant. This finding is in accordance with recent reports from some regions where the incidence of MDR $S$. typhi isolates appeared to have decreased. ${ }^{22-}$ 24 
Table.1 Salmonella Serotypes Isolated during the Study Period

\begin{tabular}{|l|l|l|}
\hline Salmonella Serotypes & Total(38) & Percentage \\
\hline Salmonella typhi & 30 & $78 \%$ \\
\hline Salmonella paratyphi A & 8 & $22 \%$ \\
\hline Other Serotypes & 0 & 0 \\
\hline
\end{tabular}

Table.2 Percentage of Salmonella typhi Resistant to Antimicrobials

\begin{tabular}{|l|l|}
\hline Antimicrobial & Percentage resistance \\
\hline Ampicillin & 66.7 \\
\hline Azithromycin & 14.2 \\
\hline Chloramphenicol & 10. \\
\hline Cefotaxime & 10.32 \\
\hline Ciprofloxacin & 13.3 \\
\hline Cotrimoxazole & 26.7 \\
\hline Cefepime & 8.32 \\
\hline Levofloxacin & 4.1 \\
\hline Nalidixic acid & 96.7 \\
\hline Cefixime & 9.75 \\
\hline
\end{tabular}

The study a underlines the importance of drugs like $3^{\text {rd }}$ generation cephalosporins and azithromycin which still have low resistance to S.typhi for treating MDR and ciprofloxacin resistant cases in this region. Emphasis is laid on the sparing use of these drugs for the treatment of uncomplicated typhoid. These should be used only if the first and second line antibiotics fail to evoke a satisfactory response.

In conclusion, the study indicated that the most Dominant serotype is S.Typhi Followed by Salmonella paratyphi A which has implications on vaccination strategy in the region. The rates of Salmonella paratyphi A have remained more or less constant over a decade when compared with previous studies.

Also, the findings of the present study indicated that the first line antibiotics may still have a role to play in the treatment of typhoid fever. Further, the usefulness of nalidixic acid as a screening test for detecting reduced susceptibility to ciprofloxacin is emphasized. A simple disk diffusion test can rapidly provide information that can predict treatment failures, and obviates the need for the more time consuming dilution tests. With ceftriaxone and Azithromycin emerging as the sole defense against NARST strains, physicians should be advised against using this drug empirically, and it should be instituted only in the event of nonresponsiveness to ciprofloxacin. The resistance to cephalosporins have increased over a decade and therefore necessary steps to be taken for its judicious dose.

The recent emergence of resistance to Nalidixic acid thereby leading to decreased susceptibility to flouroquinolones, suggests that their widespread and indiscriminate use in primary care settings should be restricted. Since in our study the bacterium is still sensitive to traditonal first-line drugs (chloramphenicol, trimethoprimsulfamethoxazole), these remain appropriate 
for the treatment of typhoid fever. They are inexpensive, widely available and rarely associated with side-effects.

Also the Usefulness of nalidixic acid as a screening test for predicting decreased resistance to other fluoroquinolones need to be emphasized and should be used by all laboratories in order to predict treatment failures.

The use of ceftriaxone and other third and 4th generation cephalosporins should be used only in case of non responsiveness to fluoroquinolones.

\section{References}

1.Miller SI, Pegues DA, Mandell GL, Bennet JE, Dolin R.1998; Principles and Practice of Infectious Disease. 4th edn. New York: Churchill Livingstone; Salmonella species, including Salmonella typhi; pp. 2344-2373.

2. Ivanoff B.1995; Typhoid fever, global situation and WHO recommendations. Southeast Asian Journal of Tropical Medicine and Public Health;26:1-6. (Suppl 2):

3. Jesudason MV, John TJ.1992: Plasmid mediated multidrug resistance in Salmonella typhi. Indian J Med Res ; 95 : 66-7.

4. Butt T, Ahmad RN, Mahmood A, Zaidi S.2003; Ciprofloxacin treatment failure in typhoid fever case, Pakistan. Emerg Infect Dis ; 9 : 1621-2

5.Jesudason MV, John TJ.1992; Plasmid mediated multidrug resistance in Salmonella typhi. Ind J Med Res 95: 667.

6. Mourad AS, Matwally M, Nour Ei Deen A, et al. (1993) Multiple drug-resistant Salmonella typhi. Clin Infect Dis 17: 135-6. 6

7.Agarwal SC (1962) Chloramphenicol resistance of Salmonella species in India, 1956-61. Bull Wld Hlth Orgn 17:
331-5. 7

8.Threfall El,Skinner JA.2001; Wardlr. Detection of decreased in vitro susceptibility to ciprofloxacin in salmonella enterica serotypes typhi and paratyphi A(JAntimicrob Chemother. 2001; 48:740-741.

9. Pary CM,2004;The treatment of multi drug resistant and nalidixic acid resistant typhoid fever in viet nam.Trans $\mathrm{R}$ sOCTrp Med Hyg.2004;98:413-414

10.RahmanM,Ahmad A,Shoma S.2002; decline in epidemic of multi drug resistant Salmonella typhi is not associated with increased incidence of antibiotic susceptible strain in Bangladesh.Epidemiol Infect;129:29-34

11.Sanghavi SK, Mane MP, Niphadkar KB.1999; Multidrug resistance in Salmonella serotypes. Indian J Med Microbiol 1999; 17 : 88-90.

12. Chande C, Shrikhande S, Kapale S, Agrawal S, Fule RP;2002. Change in antimicrobial resistance pattern of Salmonella typhi in central India. Indian J Med Res 2002; $115:$ 248-50.

13. Saha MR, Dutta P, Niyogi SK, Dutta S, Mitra U, Ramamurthy T, et al.2002; Decreasing trend in the occurrence of Salmonella enterica serotype Typhi amongst hospitalized children in Kolkata, India during 1990-2000. Indian J Med Res ; 115 : 46-8.

14..Background document: The diagnosis,treatment and prevention of typhoid fever: World Health Organizatio;21-22:May2003.

15.Saha SK, Talukder SY, Islam M, Saha S.1999; A highly ceftriaxoneresistant Salmonella typhi in Bangladesh. Pediatr Infect Dis J; $18: 387$.

16.Bhutta ZA, Khan I, Molla AM. 1994;Therapy of multidrug resistant typhoidal salmonellosis in childhood: a randomized controlled comparison of therapy with oral cefixime versus IV ceftriaxone. The Pediatric Infectious Disease Journal; 13: 990-4.

17. Girgis NI, Sutan Y, Hammad O, Farid 
Z.1995; Comparison of the efficacy, safety and cost of cefixime, ceftriaxone and aztreonam in the treatment of multidrug resistant Salmonella typhi septicemia in children. The Pediatric Infectious Disease Journal ; 14: 603-5.

18. Girgis NI, Tribble DR, Sultan Y, Farid Z.1995; Short course chemotherapy with cefixime in children with multidrug resistant Salmonella typhi septicemia. Journal of Tropical Pediatrics ; 41: 3645.

19. Madhulika.U, HarishB.N. \& ParijaS.C.2004.Current pattern in antimicrobial susceptibility of Salmonella typhi. Isolates in Pondicherry Indian J Med Res 120;111114

20. Phoung CXT, Kneen R, Anh NT, Luat TD, White NJ, Parry CM, Dong Nai.1999; Paediatric Center Typhoid Study Group. A comparative study of ofloxacin and cefixime for treatment of typhoid fever in children. The Pediatric Infectious Disease Journal;18: 245-8.

21. Chinh NT, Parry CM, Ly NT, et al.2000; A randomised controlled comparison of azithromycin and ofloxacin for multidrug-resistant and nalidixic acid resistant enteric fever. Antimicrobial Agents and Chemotherapy; 44: 1855-9.

22.Nath G, Tikoo A, Manocha H, Tripathi AK, Gulati AK. 2003; Drug resistance in Salmonella typhi in North India with special reference to ciprofloxacin; J Antimicrobial Chemother 46: 145-53.

23. Tankhiwale SS, Agrawal G, Jalgaonkar SV.2003; A Preliminary Report on
Current Antibiogram of Salmonella enterica serotype typhi in Nagpur. Ind J Med Microbiol 21: 292

24. Rodrigues C, Shennai S, Mehta A.2003; Enteric fever in Mumbai, India: the good news and the bad news. ClinI nfect Dis. 36: 535.

25.Sood S, et al.1999; Paratyphoid fever in India: an emerging problem. Emerging Infectious Diseases ; 5: 483-484.

26. Tankhiwale SS, Agrawal G, Jalgaonkar SV. An unusually high occurrence of Salmonella enteric

27. Mohanty S, Renuka K, Sood S, Das BK and Kapil A.2006: Antibiogram pattern and seasonality of Salmonella serotypes in a North Indian tertiary care hospitalEpidemiol. Infect. (2006), 134, 961-966.

28.Gautam V, et al.2002 ;Sensitivity pattern of Salmonella serotypes in Northern India. Brazilian Journal of Infectious Diseases; 6: 281-287.

29. Chandel DS, Chaudhry R, Dhawan B, Pandey A, Dey AB. 2000; Drugresistant Salmonella enterica serotype Paratyphi A in India. Emerg Infect Dis;6:420-1.

30. Brown NM, Millar MR, Frost JA, Rowe B.1994; Ciprofloxacin resistance in Salmonella paratyphi A. J Antimicrob Chemother ;33: 1258-9.

31. Harish BN, Madhulika U, Parija SC. 2004Isolated high-level ciprofloxacin resistance in Salmonella enterica subsp. enterica serotype Paratyphi A. J Med Microbiol;53: 819.

\section{How to cite this article:}

Ruchi Girotra, Reetika Dawar, Ruby naz, Sunil Garg and Rakesh Gupta. 2016. Prevalence of Salmonella serotypes and antibiogram of Salmonella typhi in a Tertiary Care Hospital in NCR Region, India. Int.J.Curr.Microbiol.App.Sci. 5(4): 803-810. doi: http://dx.doi.org/10.20546/ijcmas.2016.504.092 